\title{
The combined expression of Semaphorin4D and PlexinB1 predicts disease recurrence in colorectal cancer
}

\author{
Tetsuro Ikeya*, Kiyoshi Maeda, Hisashi Nagahara, Masatsune Shibutani, Yasuhito Iseki and Kosei Hirakawa
}

\begin{abstract}
Background: Binding to Sema4D and PlexinB1 induce angiogenesis and invasive growth in colorectal cancer (CRC). The expression of Semaphorin4D (Sema4D) and PlexinB1 has been shown to be related to the prognosis of patients with various malignancies. However, the correlation between the expression of Sema4D and PlexinB1 and the relapse-free survival in patients with colorectal cancer remains controversial.

Methods: The study population included patients who underwent surgery for colorectal cancer $(n=226)$. The expression of Sema4D and PlexinB1 were analyzed by immunohistochemistry in tissue of stage I, II, and III colon cancers.

Results: The immunohistochemical staining of colorectal cancer tissue specimens revealed that 95 (42\%) and 105 $(46.4 \%)$ of the specimens were positive for Sema4D and PlexinB1. The expression of Sema4D and PlexinB1 respectively were both found to be significantly related to stage, depth of tumor invasion, lymph node metastasis, lymphatic invasion, and venous invasion, respectively. Sixty-three patients (27.9\%) expressed both Sema4D and PlexinB1. The positive expression of both Sema4D and PlexinB1 was found to be an independent risk factor for a worse survival (HR 1.079, Cl 1.013-2.868; $P=0.044)$.

Conclusion: The combination of Sema4D and PlexinB1 protein detected by immunohistochemistry was therefore useful for predicting disease recurrence in CRC patients.
\end{abstract}

Keywords: Colorectal cancer, Semaphorin4D, PlexinB1, Recurrence

\section{Background}

Worldwide, colorectal cancer (CRC) is the second highest cause of deaths among females and the third highest cause of among males with malignant neoplasms. There are 1.4 million new cases of CRC worldwide each year; 700,000 of these patients will die from CRC. With the number of CRC patients increasing each year, CRC will become the world's most important malignancy [1].

Surgical resection is performed in most patients in whom CRC is detected. However, disease recurrence occurs in $20-25 \%$ of patients after a curative operation [2]. In spite of treatment, most patients with recurrent CRC progress to death within a relatively short period of time. In the clinical setting, it is important to prevent

\footnotetext{
* Correspondence: tetsu_47@msn.com

Department of Surgical Oncology, Osaka City University Graduate School of Medicine, Abeno-ku, Osaka, Japan
}

recurrence to prolong survival. It is therefore helpful to understand the risk factors for recurrence. Shibutani et al. reported that the combination of the preoperative level of CEA and CA19-9 was a useful biomarker for recurrence in CRC patients after a curative operation [3]. The microsatellite instability (MSI) status has been reported to be an independent prognostic predictor of time to recurrence [4].

In this study, we focused on the Semaphorin4D (SematD) proteins. Semaphorins are a large family of se linked proteins which contain a phylogenetically conserved extracellular "sema" domain. They are classified into eight classes, of which 3 to 7 contain vertebrate semaphorins $[5,6]$. Sema4D is a transmembrane protein. Through shedding by membrane type 1-matrix metalloproteinase 1 (MT1-MMP), it transforms into a soluble 
form, which mainly binds to PlexinB1 [7]. PlexinB1 is single-pass transmembrane receptor for Sema4D, which is mainly expressed on endothelial cells and epithelial cells [5].

Recently, the role of Sema4D, via interaction with PlexinB1, in activities such as tumor angiogenesis and invasive growth have been discussed in relation to various types of tumors [8-10]. Thus, in the present study we investigated the expression of Sema4D and PlexinB1 in CRC tissue specimens and assessed their association with various clinicopathological factors. Finally, we clarified the potential of these variables as risk factors for CRC recurrence.

\section{Methods}

\section{Patients}

The study population included patients who underwent surgery for colorectal cancer between 2008 and 2011 at the Department of Surgical Oncology, Osaka City University Graduate School of Medicine, Japan. Patients who received preoperative chemotherapy were excluded from the analysis. The patients consisted of 124 males and 102 females, with a median age of 66 years (range: 21-91 years). The clinicopathological classification was determined according to the TNM classification of malignant tumors, as described by the International Union Against Cancer (UICC) [11]. The tumor stages of the patients were graded as follows: stage I $(n=58)$, stage II $(n=57)$, and stage III $(n=111)$.

\section{The immunohistochemical analysis}

All tissues were fixed in $10 \%$ formalin immediately after surgical resection and $6-\mu \mathrm{m}$-thick specimens were embedded in paraffin. The immunohistochemical determination of the Sema4D and PlexinB1 levels in the colorectal cancer cells was carried out according to the manufacturer's instructions. In brief, the slides were deparaffinized in xylene and hydrated in decreasing concentrations of ethyl alcohol. The sections were then deparaffinized and incubated with $3 \%$ hydrogen peroxide in methanol for 15 min to block endogenous peroxidase activity. The tissues were subsequently heated for $10 \mathrm{~min}$ at $105^{\circ} \mathrm{C}$ by autoclaving in Target Retrieval Solution (Dako, Carpinteria, CA, USA). The sections were then washed in phosphate-buffered saline (PBS) and incubated in $10 \%$ normal rabbit serum for $10 \mathrm{~min}$ to reduce nonspecific antibody binding. The specimens were incubated with antibodies to Sema4D for $1 \mathrm{~h}$ at roomtemperature and antibodies to PlexinB1 overnight at $4{ }^{\circ} \mathrm{C}$. They were then washed twice with PBS. The primary antibodies used for the immunohistochemical detection of anti-Sema4D and anti-PlexinB1 were Rabbit polyclonal antibody to Sema4D (SIGMA-Aldrich Ltd, Poole, UK, HPA015662, 1:150) and Rabbit polyclonal antibody to
PlexinB1 (SIGMA-Aldrich Ltd, Poole, UK, HPA040586, 1:100). The sections were incubated with biotinylated rabbit anti-goat immunoglobulin $\mathrm{G}$ for $10 \mathrm{~min}$, then washed twice with PBS. The slides were then treated with peroxidase-conjugated streptavidin reagent for $5 \mathrm{~min}$ and washed twice with PBS. Finally, the slides were incubated with diaminobenzidine (DAB) kit (Histofine SAB-PO Kit; Nichirei, Tokyo, Japan) for $180 \mathrm{~s}$ for Sema4D antibodies, and $150 \mathrm{~s}$ for PlexinB1 antibodies, then counterstained with Mayer's hematoxylin and mounted.

\section{The evaluation of immunostaining for Sema4D}

We counted the total number of infiltrating inflammatory cells in the tumor stroma in three independent high-power fields $(\times 400)$ for each tissue sample. The positive Sema4D staining of inflammatory cells was observed in the tumor stroma (Fig. 1). We then calculated the percentage of Sema4D-positive cells among the total number of inflammatory cells. The specimens were then divided into three grades, according to the degree of positivity as follows: grade 1 ( $0-25 \%$ positive), grade 2 (26-50 \% positive) and grade 3 (51-100\% positive). For
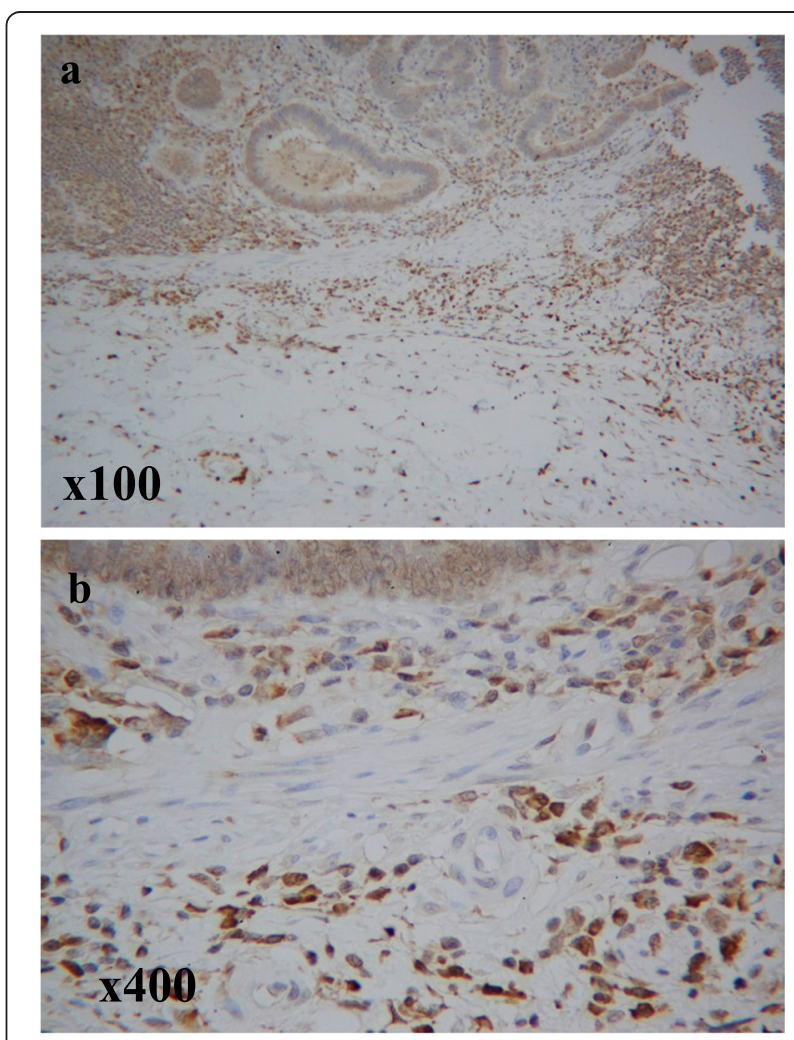

Fig. 1 Immunohistochemical staining for Semaphorin4D. The immunohistochemical evaluation of Sema4D-positive cells in colorectal cancer specimens. The positive staining of inflammatory cells was observed in the tumor stroma. a The photograph was taken at a magnification of $\times 100, \mathbf{b}$ The photograph was taken at a magnification of $\times 400$ 
the statistical analyses, grades 1 and 2 were defined as negative, and grade 3 was defined as positive [9].

\section{The evaluation of immunostaining for PlexinB1}

Positive PlexinB1 staining of the tumor gland was observed in the cytoplasm of cancer cells (Fig. 2). The staining intensity of epithelial tumor cells (in comparison to non-tumor cells) was scored as follows: 0 (no staining), 1 (weak staining), 2 (moderate staining) and 3 (strong staining). For the statistical analyses, scores of 0 or 1 were defined as negative, and a score of 2 or 3 was defined as positive [9].

\section{Statistical analysis}

The associations between the expression of Sema4D and PlexinB1 and various clinicopathological factors were assessed using the $\chi^{2}$ test or Fisher's exact test. To investigate the associations between relapse-free survival and various clinicopathological factors, a univariate survival analysis was performed using the Kaplan-Meier method, and the differences were evaluated using the log-rank test. A multivariate survival analysis was performed
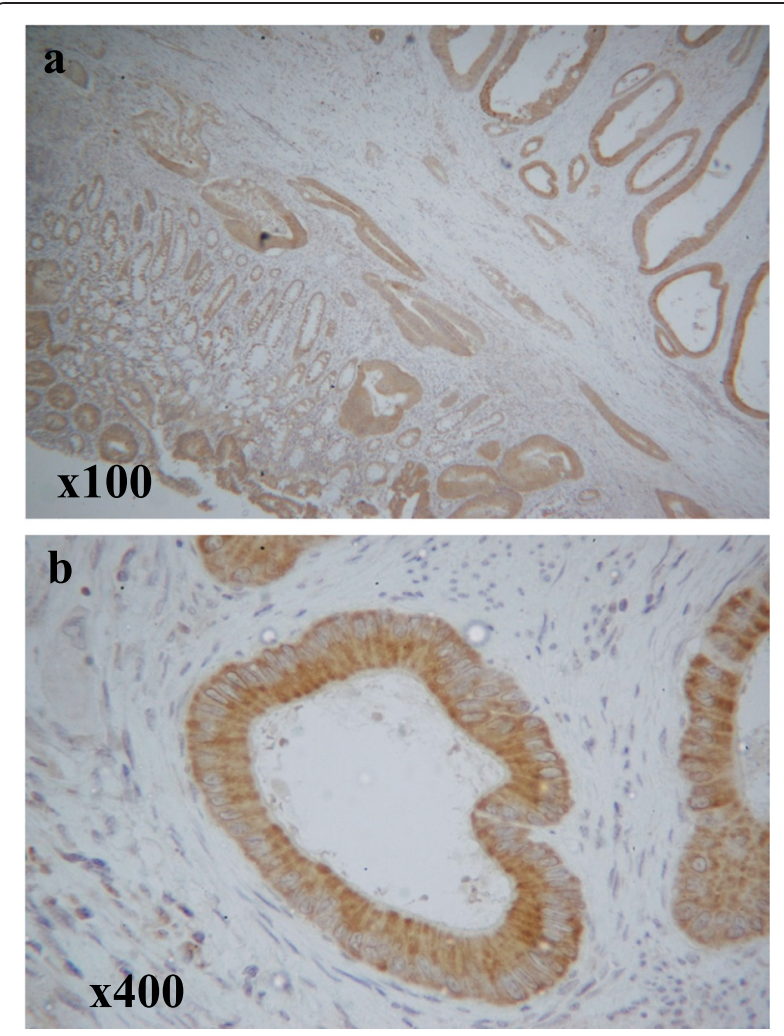

Fig. 2 Immunohistochemical staining for PlexinB1. The immunohistochemical evaluation of PlexinB1 positive cells in colorectal cancer. The positive staining of the tumor gland was observed in the cytoplasm of cancer cells. a The photograph was taken at a magnification of $\times 100, \mathbf{b}$ The photograph was taken at a magnification of $\times 400$ using Cox's proportional-hazard model. Hazard ratios and $95 \%$ confidence intervals were used to measure associations. The JMP 10 software program (SAS Institute Inc., Cary, NC, USA) was used for all of the statistical analyses. $P$ values of $<0.05$ were considered to be statistically significant.

\section{Results}

The correlations between Sema4D and PlexinB1 expression and the clinicopathological findings

The immunohistochemical staining of colorectal cancer tissue specimens revealed that 95 (42\%) and 105 (46.4\%) of the specimens were positive for Sema4D and Plexin B1, respectively. The expression of Sema4D was significantly related to stage, the depth of tumor invasion, lymph node metastasis, lymphatic invasion, and venous invasion (Table 1). Age, gender and histology did not significantly affect Sema4D expression. The expression of PlexinB1 was significantly related to stage, depth of tumor invasion, lymph node metastasis, lymphatic invasion, and venous invasion. There were no significant differences among the other factors.

\section{The correlations between Sema4D and PlexinB1 expression}

Among 85 tumors which were found to be positive for Sema4D, 63(74.1 \%) were positive for PlexinB1. On the other hand, among the 131 tumors which found to be negative for Sema4D, 42(32 \%) were negative for PlexinB1. There was a significant correlation between the expression of Sema4D and PlexinB1 $(p<0.001$, Table 2).

The correlations between positivity for both Sema4D and PlexinB1 and the clinicopathological findings

Sixty-three patients $(27.9 \%)$ expressed both Sema4D and PlexinB1. There were no significant differences in the amount of infiltrated inflammatory cells around the tumor stroma between the positive Sema4D/PlexinB1 group and the negative Sema4D/PlexinB1 group ( $p=$ 0.539 ). We examined the correlations between the positive expression of both Sema4D and PlexinB1 and the clinicopathological findings. The expression of both Sema4D and PlexinB1 was found to be correlated with all of the clinicopathological factors that were examined, with the exception of age, gender and histology (Table 3). In addition, we evaluated the presence of any correlation between the treatment as an adjuvant chemotherapy and the expression of Sema4D and PlexinB1. Adjuvant chemotherapy was administered to patients who belonged to the high risk groups with stage II and stage III disease, except for patients who rejected any further treatment and those who demonstrated a poor performance status. As a result, no significant differences were observed between the adjuvant chemotherapy and the 
Table 1 Correlations between clinicopathological findings and the expression of Sema4D and PlexinB1

\begin{tabular}{|c|c|c|c|c|c|c|}
\hline \multirow[t]{3}{*}{ Variables } & \multicolumn{2}{|c|}{ Sema4D expression } & \multirow[t]{3}{*}{$p$ value } & \multicolumn{2}{|c|}{ PlexinB1 expression } & \multirow[t]{3}{*}{$p$ value } \\
\hline & Positive & Negative & & Positive & Negative & \\
\hline & $(n=95)$ & $(n=131)$ & & $(n=105)$ & $(n=121)$ & \\
\hline \multicolumn{7}{|l|}{ Age (years) } \\
\hline Mean \pm SD & $65.2 \pm 12.3$ & $67.2 \pm 10.5$ & 0.426 & $67.6 \pm 11.0$ & $65.8 \pm 11.5$ & 0.23 \\
\hline \multicolumn{7}{|l|}{ Gender } \\
\hline Male & $51(53.7)$ & 73(55.7) & 0.76 & $56(53.3)$ & 68 & 0.666 \\
\hline Female & $44(46.3)$ & $58(44.3)$ & & 49(46.7) & 53 & \\
\hline \multicolumn{7}{|l|}{ Stage } \\
\hline I & 15(15.8) & $43(32.8)$ & 0.001 & $16(15.2)$ & 42 & $<0.001$ \\
\hline$\|$ & $21(22.1)$ & $36(27.5)$ & & 19(18.1) & 38 & \\
\hline III & $59(62.1)$ & $52(36.7)$ & & $70(66.7)$ & 41 & \\
\hline \multicolumn{7}{|l|}{ Histology } \\
\hline Well/mod & $82(86.3)$ & 120(91.6) & 0.206 & 91 & 111 & 0.217 \\
\hline Other & 13(13.7) & $11(8.4)$ & & 14 & 10 & \\
\hline \multicolumn{7}{|c|}{ Depth of tumor invasion } \\
\hline $\mathrm{T} 1 / \mathrm{T} 2$ & 18(18.9) & $50(38.2)$ & 0.001 & 20 & 48 & 0.006 \\
\hline $\mathrm{T} 3 / \mathrm{T} 4$ & $77(81.1)$ & $81(61.8)$ & & 85 & 73 & \\
\hline \multicolumn{7}{|c|}{ Lymph node metastasis } \\
\hline negative & 37(38.9) & $78(59.5)$ & 0.001 & 35 & 79 & $<0.001$ \\
\hline positive & $58(61.1)$ & $53(40.5)$ & & 69 & 42 & \\
\hline \multicolumn{7}{|c|}{ Lymphatic invasion } \\
\hline Negative & 18(18.9) & $66(50.4)$ & $<0.001$ & 21 & 57 & $<0.001$ \\
\hline Positive & 77(81.1) & $65(49.6)$ & & 84 & 64 & \\
\hline \multicolumn{7}{|l|}{ Venus invasion } \\
\hline Negative & 70(73.7) & 117(89.3) & $<0.001$ & 76 & 111 & $<0.001$ \\
\hline Positive & $25(26.3)$ & 14(10.7) & & 29 & 10 & \\
\hline
\end{tabular}

Sema4D semaphorin 4D. Other poorly-differnetiated, mucinous-type, small-cell, signet-cell

combination of Sema4D and PlexinB1 expression at each stage (Table 4).

\section{Measurement of the overall survival and relapse-free survival}

The patients of both Sema4D and PlexinB1 positive groups exhibited a worse prognosis compared to the others $(p<0.001$, Fig. 3$)$. In the univariate analysis, histology, the depth of tumor invasion, lymph node

Table 2 Correlation between Sema4D and PlexinB1 expression in colorectal cancer

\begin{tabular}{lll}
\hline & $\begin{array}{l}\text { Sema4D positive } \\
(n=85)\end{array}$ & $\begin{array}{l}\text { Sema4D negative } \\
(n=131)\end{array}$ \\
\hline PlexinB1 positive & $63(27.9)$ & $42(18.6)$ \\
$(n=105)$ & & \\
PlexinB1 negative & $32(14.1)$ & $89(39.4)$ \\
$(n=121)$ & & \\
\hline Sema4D semaphorin 4D & &
\end{tabular}

Sema4D semaphorin 4D metastasis, lymphatic invasion, venous invasion and positivity for both Sema4D and PlexinB1 were found to be significantly associated with overall survival (Table 5). However, a multivariate analysis demonstrated that the depth of tumor invasion (HR 2.692, CI 1.0229.317; $P=0.044$ ), lymph node metastasis (HR 2.304, CI 1.138-5.069; $P=0.019$ ), and the positive expression of both Sema4D and PlexinB1 (HR 1.681, CI 1.0042.819; $P=0.047$ ) were an independent risk factors for worse survival (Table 5). The recurrence rate was $46 \%$ $(29 / 63)$ in patients who positively expressed both Sema4D and PlexinB1, and $16.6 \%(27 / 163)$ in the other patients; this amounted to a significant difference $(p<0.001)$. The relapse-free survival (RFS) of the patients who positively expressed both Sema4D and PlexinB1was significantly worse than that of other patients $(p<0.001$, Fig. 4$)$. In the univariate analysis, histology, the depth of tumor invasion, lymph node metastasis, lymphatic invasion, venous invasion and positivity for both Sema4D and PlexinB1 were found to be significantly associated with RFS (Table 6). 
Table 3 Correlations between clinicopathological findings and the combination of Sema4D and PlexinB1 expresion

\begin{tabular}{|c|c|c|c|}
\hline \multirow[t]{3}{*}{ Variables } & \multicolumn{2}{|c|}{ Sema4D and PlexinB1 expression } & \multirow[t]{3}{*}{$p$ value } \\
\hline & Both positive & Others & \\
\hline & $(n=63)$ & $(n=163)$ & \\
\hline \multicolumn{4}{|l|}{ Age (years) } \\
\hline Mean \pm SD & $68.1 \pm 10.2$ & $66.0 \pm 11.7$ & 0.351 \\
\hline \multicolumn{4}{|l|}{ Gender } \\
\hline Male & 35 & 89 & 0.897 \\
\hline Female & 28 & 74 & \\
\hline \multicolumn{4}{|l|}{ Stage } \\
\hline । & 7 & 51 & $<0.001$ \\
\hline$\|$ & 11 & 46 & \\
\hline III & 45 & 66 & \\
\hline \multicolumn{4}{|l|}{ Histology } \\
\hline Well/mod & 53 & 149 & 0.123 \\
\hline Other & 10 & 14 & \\
\hline \multicolumn{4}{|c|}{ Depth of tumor invasion } \\
\hline $\mathrm{T} 1 / \mathrm{T} 2$ & 10 & 58 & 0.002 \\
\hline $\mathrm{T} 3 / \mathrm{T} 4$ & 53 & 105 & \\
\hline \multicolumn{4}{|c|}{ Lymph node metastasis } \\
\hline negative & 18 & 96 & $<0.001$ \\
\hline positive & 45 & 67 & \\
\hline \multicolumn{4}{|c|}{ Lymphatic invasion } \\
\hline Negative & 9 & 69 & $<0.001$ \\
\hline Positive & 54 & 94 & \\
\hline \multicolumn{4}{|l|}{ Venus invasion } \\
\hline Negative & 41 & 146 & $<0.001$ \\
\hline Positive & 22 & 17 & \\
\hline
\end{tabular}

Sema4D semaphorin 4D. Other poorly-differnetiated, mucinous-type, small-cell, signet-cell

However, a multivariate analysis demonstrated that lymph node metastasis (HR 2.783, CI 1.425-5.822; $P=0.002$ ), and the positive expression of both Sema4D and PlexinB1 (HR 1.079, CI 1.013-2.868; $P=0.044$ ) were an independent risk factors for worse survival (Table 6).

\section{Discussion}

The expression of Sema4D and PlexinB1 has been shown to be related to the prognosis of patients with various malignancies. Wang et al., reported the expression of Sema4D to be a novel indicator of a poor prognosis in CRC patients [8]. Kato et al., reported that the positive expression of both Sema4D and PlexinB1 was significantly correlated with worse survival in patients with pancreatic cancer [9].

On the other hand, it was reported that the decreased expression of Sema4D and PlexinB1 was associated with local recurrence and poor prognosis in breast cancer
Table 4 Correlations between adjuvant chemotherapy and the combination of Sema4D and PlexinB1 expression

\begin{tabular}{|c|c|c|c|c|}
\hline \multirow[t]{3}{*}{ Variables } & \multirow{3}{*}{$\begin{array}{l}\text { Total } \\
(n=226)\end{array}$} & \multicolumn{2}{|c|}{$\begin{array}{l}\text { Sema4D and PlexinB1 } \\
\text { expression }\end{array}$} & \multirow[t]{3}{*}{$p$ value } \\
\hline & & Both positive & Others & \\
\hline & & $(n=63)$ & $(n=163)$ & \\
\hline \multicolumn{5}{|l|}{ Stage I } \\
\hline \multicolumn{5}{|c|}{ Adjuvant chemotherapy } \\
\hline Treated & $0(0)$ & $0(0)$ & $0(0)$ & - \\
\hline Untreated & $58(100)$ & $7(100)$ & $51(100)$ & \\
\hline \multicolumn{5}{|l|}{ stage II } \\
\hline \multicolumn{5}{|c|}{ Adjuvant chemotherapy } \\
\hline Treated & $13(22.8)$ & $3(27.3)$ & $10(21.7)$ & 0.698 \\
\hline Untreated & $44(77.2)$ & $8(72.7)$ & $36(78.3)$ & \\
\hline \multicolumn{5}{|l|}{ Stage III } \\
\hline \multicolumn{5}{|c|}{ Adjuvant chemotherapy } \\
\hline Treated & $81(73)$ & $35(77.8)$ & $46(69.7)$ & 0.106 \\
\hline Untreated & $30(27)$ & $10(22.2)$ & $20(30.3)$ & \\
\hline
\end{tabular}

Sema4D semaphorin 4D

[10]. Although, the expression of Sema4D and PlexinB1 have been investigated in some solid malignancies, the correlation between the expression of these factors and the prognosis of patients with malignant tumors remains controversial.

Angiogenesis and invasive growth are induced through binding to Sema4D and PlexinB1. Consequently, the Sema4D and PlexinB1 positivity increases the possibility of relapse. Two pathways downstream to PlexinB1 have been reported to be mechanisms which underlie angiogenesis and invasive growth. The first mechanism is the transactivation of the tyrosine kinase activity of Met [12], a tyrosine kinase receptor which mediates invasive growth [13], the other is the activation of small GTPase Ras homolog gene family member A (RhoA) [14], Class

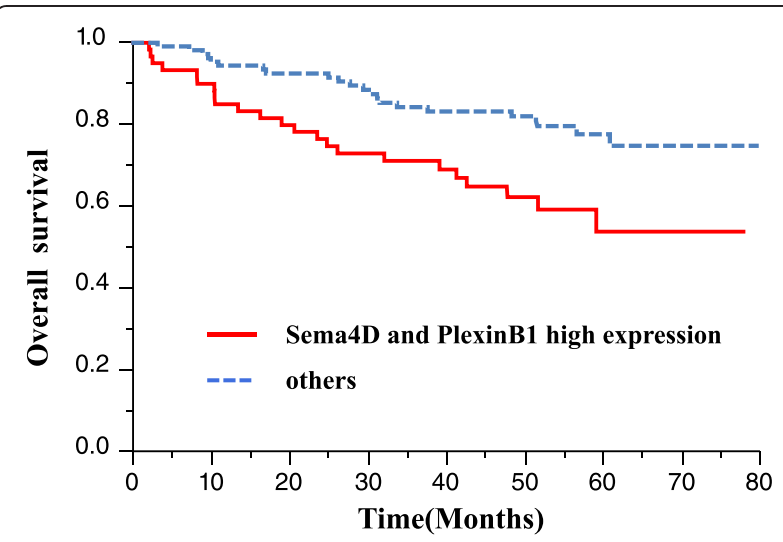

Fig. 3 Kaplan-Meier estimates of over all survival among colorectal cancer patients with the positive expression of both Sema4D and PlexinB1 
Table 5 The results of univariate and multivariate analyses of the expression of Sema4D and PlexinB1, clinicopathological findings and overall survival in the patients with colorectal cancer

\begin{tabular}{|c|c|c|c|c|c|c|}
\hline \multirow[b]{2}{*}{ Variables } & \multicolumn{3}{|c|}{ Univariate analysis } & \multicolumn{3}{|c|}{ Multivariate analysis } \\
\hline & Hazard ratio & $95 \% \mathrm{Cl}$ & $p$ value & Hazard ratio & $95 \% \mathrm{Cl}$ & $p$ value \\
\hline Gender & 0.928 & $0.574-1.509$ & 0.762 & & & \\
\hline \multicolumn{7}{|l|}{ Male vs. Female } \\
\hline Age & 1.365 & $0.841-2.207$ & 0.205 & & & \\
\hline \multicolumn{7}{|l|}{$\geqq 70$ vs. $<70$} \\
\hline Histology & 2.666 & $1.391-4.373$ & 0.004 & 1.758 & $0.901-3.205$ & 0.094 \\
\hline \multicolumn{7}{|l|}{ Other vs. well/mod } \\
\hline Depth of tumor invasion & 4.574 & $2.032-13.08$ & $<0.001$ & 2.692 & $1.022-9.317$ & 0.044 \\
\hline \multicolumn{7}{|l|}{ T3/T4 vs.T1/T2 } \\
\hline Lymph node metastasis & 4.469 & $2.432-9.017$ & $<0.001$ & 2.304 & $1.138-5.069$ & 0.019 \\
\hline \multicolumn{7}{|l|}{ Positive vs. negative } \\
\hline Lymphatic invasion & 3.108 & $1.622-6.725$ & $<0.001$ & 1.318 & $0.603-3.235$ & 0.505 \\
\hline \multicolumn{7}{|l|}{ Positive vs. negative } \\
\hline Venus invasion & 2.6 & $1.554-4.25$ & $<0.001$ & 1.409 & $0.813-2.394$ & 0.216 \\
\hline \multicolumn{7}{|l|}{ Positive vs. negative } \\
\hline Sema4D and PlexinB1 expression & 2.567 & $1.587-4.158$ & $<0.001$ & 1.681 & $1.004-2.819$ & 0.047 \\
\hline Both positive vs. others & & & & & & \\
\hline
\end{tabular}

Sema4D semaphorin 4D. Other poorly-differnetiated, mucinous-type, small-cell, signet-cell. Cl confidence interval

IV semaphorins promote angiogenesis by stimulating Rho-initiated pathways through Plexin-B, and the phosphorylation of MAPK and Akt [15]. The interaction of these signal cascades contributes to the progression of cancer. As a result, the combination of the two mechanisms reflects tumor progression and a worse RFS.

Sema4D can bind to several receptors and induce various effects [16]. CD72, a member of the C-type lectin family, is a low-affinity Sema4D receptor that is expressed on immune cells, such as B cells, dendritic cells, macrophages and mast cells. The interaction

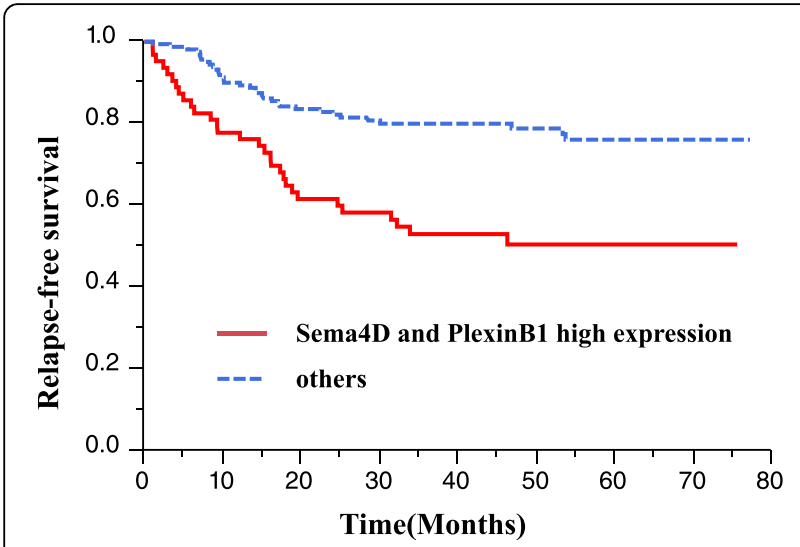

Fig. 4 Kaplan-Meier estimates of relapse-free survival among colorectal cancer patients with the positive expression of both Sema4D and PlexinB1 between the immune cells promotes the aggregation and survival of B cells, and enhances the activation of B cells during antibody production [6]. The high-affinity Sema4D receptor plexinB1 is mainly expressed on endothelial cells and epithelial cells and promotes their motility $[7,17]$. Plexin B2 receptors have a low-affinity for Sema4D, and are involved, with the mediation of dendritic epidermal $\mathrm{T}$ cells, in the wound healing process in the skin $[18,19]$. Thus, Sema4D receptors are located in various places and have many roles. Above all, PlexinB1 receptors have a high affinity for Sema4D, and contribute to tumor progression. As a result, it is a factor that may cause recurrence.

Kato reported the cells expressing Sema4D in the tumor stroma of pancreatic cancer to be tumorinfiltrating lymphocytes(mainly $\mathrm{T}$ cells and $\mathrm{B}$ cells) [9]. In this study, we could not clearly elucidate the cell in which Sema4D is expressed, although, we consider that Sema4D is also expressed in the T cells and B cells in the tumor stroma of colon cancer. It has recently been hypothesized that Sema4D is involved in the regulation of the immune response in the tumor microenvironment. Evans et al. demonstrated that Sema4D creates a barrier to immune infiltration and affects the balance of regulatory and effector immune cells and signals. These immunomodulatory functions promote tumor progression [20]. Thus, there is a possibility that cancers in which Sema4D and PlexinB1 is expressed have enhanced 
Table 6 The results of univariate and multivariate analyses of the expression of Sema4D and PlexinB1, clinicopathological findings and relapse-free survival in the patients with colorectal cancer

\begin{tabular}{|c|c|c|c|c|c|c|}
\hline \multirow[b]{2}{*}{ Variables } & \multicolumn{3}{|c|}{ Univariate analysis } & \multicolumn{3}{|c|}{ Multivariate analysis } \\
\hline & Hazard ratio & $95 \% \mathrm{Cl}$ & $p$ value & Hazard ratio & $95 \% \mathrm{Cl}$ & $p$ value \\
\hline Gender & 0.966 & $0.598-1.617$ & 0.966 & & & \\
\hline \multicolumn{7}{|l|}{ Male vs. Female } \\
\hline Age & 1.508 & $0.922-2.473$ & 0.106 & & & \\
\hline \multicolumn{7}{|l|}{$\geqq 70$ vs. $<70$} \\
\hline Histology & 2.845 & $1.480-5.081$ & 0.002 & 1.743 & $0.894-3.178$ & 0.099 \\
\hline \multicolumn{7}{|l|}{ Other vs. well/mod } \\
\hline Depth of tumor invasion & 4.062 & $1.985-9.781$ & $<0.001$ & 2.12 & $0.911-5.824$ & 0.083 \\
\hline \multicolumn{7}{|l|}{ T3/T4 vs.T1/T2 } \\
\hline Lymph node metastasis & 4.871 & $2.729-9.358$ & $<0.001$ & 2.783 & $1.425-5.822$ & 0.002 \\
\hline \multicolumn{7}{|l|}{ Positive vs. negative } \\
\hline Lymphatic invasion & 3.848 & $2.001-8.344$ & $<0.001$ & 1.658 & $0.740-4.129$ & 0.227 \\
\hline \multicolumn{7}{|l|}{ Positive vs. negative } \\
\hline Venus invasion & 2.183 & $1.217-3.729$ & 0.01 & 1.049 & $0.569-1.856$ & 0.873 \\
\hline \multicolumn{7}{|l|}{ Positive vs. negative } \\
\hline Sema4D and PlexinB1 expression & 2.647 & $1.611-4.326$ & $<0.001$ & 1.079 & $1.013-2.868$ & 0.044 \\
\hline Both positive vs. others & & & & & & \\
\hline
\end{tabular}

Sema4D semaphorin 4D. Other poorly-differnetiated, mucinous-type, small-cell, signet-cell. Cl confidence interval

invasive capacity through their control of the host immune response and that they may, as a consequence, cause a relapse.

In this study, the combined expression of Sema4D and PlexinB1 was found to be an independent risk factor for disease relapse in the multivariate analysis (Table 5). Although no significant differences were observed between the adjuvant chemotherapy and the combination of Sema4D and PlexinB1 expression at each stage, the relapse-free survival of the patients who positively expressed both Sema4D and PlexinB1 was significantly worse than that of other patients (Fig. 3). As a result, the positive expression of both Sema4D and PlexinB1 was thus considered to be closely associated with recurrence. Although the evaluation of the individual expression of either Sema4D or Plexin-B1 was an indicator of malignant potential $[8,21]$, it is thought that the combination of both Sema4D and PlexinB1 is a more accurate predictor of recurrence in CRC patients.

While Sema4D and PlexinB1 may represent sensitive biomarkers for helping to select patients who are at a high risk of early relapse, our retrospective data analysis didn't support attempting to definitively link their expression to predicting patient outcomes as a function of postoperative adjuvant chemotherapy (Table 4). Therefore, further studies on the effects of Sema4D and PlexinB1 are needed for evaluating their relevance regarding selecting patients for postoperative adjuvant chemotherapy.

\section{Conclusion}

Our results indicated that the detection of the combination of Sema4D and PlexinB1 was useful for predicting disease recurrence in CRC patients. However, further studies will be necessary to more definitively investigate whether the detection of the combination of Sema4D and PlexinB1 may be useful for selecting cases in which postoperative adjuvant chemotherapy will be efficacious after curative resection.

\section{Abbreviations}

CRC, colorectal cancer; DAB, diaminobenzidine; MSI, microsatellite instability; MT1-MMP, membrane type 1-matrix metalloproteinase 1; PBS, phosphatebuffered saline; Sema4D, Semaphorin4D.

\section{Acknowledgments}

The author are independent of any commercial funder, had full access to all the data and take responsibility for the integrity of the data and analyses.

\section{Availability of data and materials}

The datasets supporting the conclusion of this article is included within articles. Any request of data and material may be sent to the corresponding author.

\section{Authors' contributions}

$\mathrm{TI}$ and $\mathrm{KM}$ designed the study and wrote protocol. TI, KM, HN, SM, YI and $\mathrm{KH}$ enrolled the patients. TI and KM were responsible for data management, statistical analysis and data interpretation. TI drafted the manuscript. All authors were involved in writing manuscript and approved the final version.

Competing interests

The authors declare that they have no competing interests. 


\section{Consent for publication}

Written informed consent for publication of their clinical details and/or clinical images was obtained from the patient.

\section{Ethics approval and consent to participate}

This study was approval by the ethics committee of Osaka City University and all the patients included in this retrospective analysis provided their written informed consent.

Received: 19 October 2015 Accepted: 18 July 2016

Published online: 25 July 2016

\section{References}

1. Globocan 2013 Colorectal cancer. Estimated cancer incidence, mortality and prevalence worldwide in 2012. http://globocan.iarc.fr/Pages/fact_sheets_ cancer.aspx.

2. Edge S, Byrd D, Compton C, Fritz A, Greene F, Trotti A. AJCC cancer staging manual. 7th ed. New York: Springer; 2010.

3. Shibutani M, Maeda K, Nagahara H, Ohtani H, Sakurai K, Toyokawa T, et al. Significance of CEA and CA19-9 combination as a prognostic indicator and for recurrence monitoring in patients with stage II colorectal cancer. Anticancer Res. 2014;34:3753-8.

4. Popat S, Hubner R, Houlston RS. Systematic review of microsatellite instability and colorectal cancer prognosis. J Clin Oncol. 2005;23:609-61.

5. Ch'ng ES, Kumanogoh A. Roles of Sema4D and Plexin-B1 in tumor progression. Mol Cancer. 2010;21:251.

6. Kumanogoh A, Watanabe C, Lee I, Wang X, Shi W, Araki H, et al. Identification of CD72 as a lymphocyte receptor for the class IV semaphorin CD100: a novel mechanism for regulating B cell signaling. Immunity. 2000;13:621-31.

7. Basile JR, Gavard J, Gutkind JS. Plexin-B1 utilizes RhoA and Rho kinase to promote the integrin-dependent activation of Akt and ERK and endothelial cell motility. J Biol Chem. 2007;282:34888-95.

8. Wang JS, Jing CQ, Shan KS, Chen YZ, Guo XB, Cao ZX, et al. Semaphorin 4D and hypoxia-inducible factor-1a overexpression is related to prognosis in colorectal carcinoma. World J Gastroenterol. 2015;21:2191-8.

9. Kato S, Kubota K, Shimamura T, Shinohara Y, Kobayashi N, Watanabe S, et al. Semaphorin 4D, a lymphocyte semaphorin, enhances tumor cell motility through binding its receptor, plexinB1, in pancreatic cancer. Cancer Sci. 2011;102:2029-37.

10. Malik MF, Ye L, Jiang WG. Reduced expression of semaphorin 4D and plexin-B in breast cancer is associated with poorer prognosis and the potential linkage with oestrogen receptor. Oncol Rep. 2015;34:1049-57.

11. Sobin L, Gospodarowicz M, Wittekind C. TNM classification of malignant tumors. 7th ed. New York: Wiley: International Union Against Cancer; 2009. p. 73-7.

12. Giordano S, Corso S, Conrotto P, Artigiani S, Gilestro G, Barberis D, et al. The semaphorin $4 \mathrm{D}$ receptor controls invasive growth by coupling with Met. Nat Cell Biol. 2002;4:720-4

13. Gentile A, Trusolino L, Comoglio PM. The Met tyrosine kinase receptor in development and cancer. Cancer Metastasis Rev. 2008;27:85-94.

14. Basile JR, Barac A, Zhu T, Guan KL, Gutkind JS. Class IV semaphorins promote angiogenesis by stimulating Rho-initiated pathways through plexin-B. Cancer Res. 2004;64:5212-24.

15. Basile JR, Holmbeck K, Bugge TH, Gutkind JS. MT1-MMP controls tumorinduced angiogenesis through the release of semaphorin 4D. J Biol Chem. 2007;282:6899-905.

16. Zhang $Y$, Liu B, Ma Y, Jin B. Sema 4D/CD100-plexin B is a multifunctional counter-receptor. Cell Mol Immunol. 2013;10:97-8.

17. Tamagnone L, Artigiani S, Chen H, He Z, Ming Gl, Song H, Chedotal A, et al. Plexins are a large family of receptors for transmembrane, secreted, and GPI anchored semaphorins in vertebrates. Cell. 1999;99:71-80.

18. Masuda K, Furuyama T, Takahara M, Fujioka S, Kurinami H, Inagaki S. Sema4D stimulates axonal outgrowth of embryonic DRG sensory neurones. Genes Cells. 2004;9:821-9.

19. Witherden DA, Watanabe M, Garijo O, Rieder SE, Sarkisyan G, Cronin J, et al. The CD100 receptor interacts with its plexin B2 ligand to regulate epidermal gammadelta T cell function. Immunity. 2012:37:314-25.
20. Evans EE, Jonason Jr AS, Bussler H, Torno S, Veeraraghavan J, Reilly C, et al. Antibody blockade of semaphorin 4D promotes immune infiltration into tumor and enhances response to other immunomodulatory therapies. Cancer Immunol Res. 2015:3:689-701.

21. Stevens $L$, McClelland L, Fricke A, Williamson M, Kuo I, Scott G. Plexin B1 suppresses c-Met in melanoma: a role for plexin B1 as a tumorsuppressor protein through regulation of c-Met. J Invest Dermatol. 2010;130:1636-45

\section{Submit your next manuscript to BioMed Central and we will help you at every step:}

- We accept pre-submission inquiries

- Our selector tool helps you to find the most relevant journal

- We provide round the clock customer support

- Convenient online submission

- Thorough peer review

- Inclusion in PubMed and all major indexing services

- Maximum visibility for your research

Submit your manuscript at www.biomedcentral.com/submit 